\title{
Performance of Candidate Serum Biomarkers for Systemic Sclerosis-Associated Interstitial Lung Disease
}

\author{
Muriel Elhai, ${ }^{1}$ Anna Maria Hoffmann-Vold, ${ }^{2}$ Jérôme Avouac, ${ }^{1}$ Sonia Pezet, ${ }^{3}$ Anne Cauvet, ${ }^{3}$ Agathe Leblond, ${ }^{3}$ \\ Håvard Fretheim, ${ }^{2}$ Torhild Garen, ${ }^{2}$ Masataka Kuwana, ${ }^{4}$ Øyvind Molberg, ${ }^{2}$ and Yannick Allanore ${ }^{1}$
}

Objective. Interstitial lung disease (ILD) in systemic sclerosis (SSc) runs a highly variable course, and prediction tools are highly desired. The aim of this study was to assess the diagnostic and prognostic performance of 4 candidate serum biomarkers for SSc-associated ILD.

Methods. Serum samples from a combined cohort of SSc patients (from Paris, France and Oslo, Norway; $\mathrm{n}=$ 427) were analyzed by enzyme-linked immunosorbent assay for concentrations of lung epithelial-derived surfactant protein D (SP-D), Krebs von den Lungen 6 glycoprotein (KL-6), CCL18, and OX40 ligand (OX40L). Lung fibrosis was measured by high-resolution computed tomography and pulmonary function tests. Associations of these candidate biomarkers with baseline disease involvement and prediction of disease progression over time (mean \pm SD follow-up $3.2 \pm 4.4$ years) were investigated.

Results. In SSc patients at baseline, serum levels of KL-6 correlated with the forced vital capacity (FVC) $(r=$ -0.317, $P<0.001)$, diffusing capacity for carbon monoxide $(r=-0.335, P<0.001)$, and extent of lung fibrosis $(r=$ $0.551, P<0.001)$. In multivariate analyses, serum levels of KL-6 and SP-D, but not CCL18 and OX40L, were associated with lung fibrosis (odds ratio [OR] 2.41, 95\% confidence interval [95\% $\mathrm{Cl}] 1.43-4.07[P=0.001]$ and OR 3.15, 95\% Cl 1.81-5.48 [ $P<0.001]$, respectively). In SSc patients with ILD at baseline, longitudinal, multivariate analyses showed that CCL18 serum levels were an independent predictor of a $>10 \%$ decrease in the FVC (hazard ratio [HR] $2.90,95 \% \mathrm{Cl} 1.25-6.73 ; P=0.014)$ and de novo development of extensive disease (HR 3.71, 95\% $\mathrm{Cl} 1.02-13.52 ; P$ $=0.048$ ). Matrix-based logistic regression models for the diagnosis and prognosis of SSc-associated ILD were constructed, and these models discriminated 3 groups of risk (mild, moderate, or high) for the diagnosis or worsening of lung fibrosis according to the serum levels of SP-D (for diagnosis) and serum levels of CCL18 (for progression of disease).

Conclusion. These results show that SP-D is a relevant diagnostic biomarker for SSc-associated ILD, whereas KL-6 could be used to assess the severity of lung fibrosis. CCL18 appears to be a potential predictive marker for progression of ILD in SSc.

\section{INTRODUCTION}

Interstitial lung disease (ILD) is common in systemic sclerosis (SSc), and despite recent advances in treatment, it remains the leading cause of death in patients with SSc (14). However, SSc-associated ILD is characterized by high heterogeneity: some patients have limited, nonprogressive

Supported by Institut Roche and the Association des Sclérodermiques de France.

${ }^{1}$ Muriel Elhai, MD, PhD, Jérôme Avouac, MD, PhD, Yannick Allanore, MD, PhD: INSERM U1016, UMR8104, Cochin Institute, Paris Descartes University, and Cochin Hospital, Paris Descartes University, Paris, France; ${ }^{2}$ Anna Maria Hoffmann-Vold, MD, PhD, Håvard Fretheim, MD, Torhild Garen, MD, PhD, Øyvind Molberg, MD, PhD: Oslo University Hospital, Oslo and Institute of Clinical Medicine, University of Oslo, Oslo, Norway; ${ }^{3}$ Sonia Pezet, Anne Cauvet, Agathe Leblond: INSERM U1016, UMR8104, Cochin Institute, Paris Descartes University, Paris, France; ${ }^{4}$ Masataka Kuwana, MD, PhD: Nippon Medical School, Tokyo, Japan. fibrosis, while others will develop extensive fibrosis that rapidly progresses to respiratory failure $(1,5)$. Until now, pulmonary function tests (PFTs) and high-resolution computed tomography (HRCT) have remained the mainstays not only for diagnosis, but also for prognosis of SSc-associated ILD, but there have been concerns regarding the potential for radiation exposure with HRCT $(6,7)$.

Drs. Elhai and Hoffmann-Vold contributed equally to this work. No potential conflicts of interest relevant to this article were reported. Address correspondence to Yannick Allanore, MD, PhD, Cochin Institute, INSERM U1016, UMR 8104, Paris Descartes University, Rheumatology A Department, Cochin Hospital, 27 Rue du Faubourg St. Jacques, 75014 Paris, France. E-mail:yannick.allanore@cch.aphp.fr. 

With the recent development of new treatments for lung fibrosis, it is critical to identify those patients who will develop lung disease at an earlier stage, and to rapidly identify those whose disease will progress to extensive lung fibrosis (8-10). Moreover, enriched populations of patients would facilitate clinical trials and the faster development of innovative therapies. To date, no validated lung functional/radiologic measures or serologic markers that would allow the prediction of progression of lung fibrosis over time in patients with SSc have been developed; such measures could help individualize the management of specific risk in each patient (11). Therefore, there is a growing interest in identifying biomarkers for the 1) diagnosis of lung fibrosis, 2) assessment of lung fibrosis severity, and 3) prognosis in patients with SSc who have already been diagnosed as having ILD (prediction of lung fibrosis worsening) (12). Four promising serum biomarkers of SSc-associated ILD have so far been suggested, including lung epithelial-derived surfactant protein D (SP-D), Krebs von den Lungen 6 glycoprotein (KL-6), CCL18, and soluble OX40 ligand (OX40L). However, results have been conflicting with regard to the sensitivity and specificity of these candidate biomarkers and their performance in the prediction of outcomes, with some limitations being attributable to small sample sizes and variability in outcome measures (12-19).

The objectives of the present study in a large cohort of patients with SSc were to assess the performance of these 4 candidate biomarkers for the 1) diagnosis of SSc-associated ILD, 2) assessment of lung fibrosis severity, and 3) prognosis (risk stratification) among SSc patients diagnosed as having lung fibrosis at baseline.

\section{PATIENTS AND METHODS}

Study cohorts. Taking advantage of our previous close collaboration with regard to SSc-associated ILD, we built a large cohort of 427 patients with SSc constituted by 2 patient populations, issued from Norway and France. In this combined cohort, all patients fulfilled the American College of Rheumatology/European League Against Rheumatism 2013 classification criteria for SSc (20). Entry criteria included requiring patients to have longitudinal data from paired PFTs and lung $\mathrm{HRCT}$, as well as baseline serum samples available for biomarker analyses. Data on demographic features, disease subset, antibodies, disease duration, smoking status, the presence of internal organ involvement, and treatments were also collected. Time of onset of SSc was defined as the appearance of the first non-Raynaud's symptom. Disease duration was defined as the time between the first non-Raynaud's symptom and blood sample collection. The follow-up period was defined as the time from baseline lung assessment to last available lung assessment.

For the Norwegian cohort, the study was approved by the Regional Committee of Health and Medical Research Ethics in
South-East Norway. All patients in the French cohort signed an informed consent form that was approved by the local institutional review boards (Comité de Protection des Personnes, Paris lle de France 3).

Serial assessment of SSc-associated ILD. In both centers, all patients with SSc are assessed by HRCT and PFTs at the time of the first visit. In the French cohort, each patient undergoes PFTsatleastannually, whereas HRCT is repeated only in cases of clinical or functional suspicion of disease progression. In the Norwegian cohort, all patients are followed up annually with HRCT and PFTs. In instances of severe ILD and any ILD that has been treated, the patients are followed up every 3 months with clinical evaluations and PFTs, and annually with HRCT.

Lung fibrosis was diagnosed using HRCT (21). Reticularpattern abnormalities and ground-glass opacities were defined as being equivalent to fibrosis, and were measured by drawing the region of interest. Paired PFTs and HRCT lung images were obtained both at baseline and at the last available follow-up visit.

The extent of fibrosis on baseline and follow-up HRCTs was measured in a manner as previously described, with results expressed as the percentage of fibrosis based on total lung volume (22). CT images were reconstructed at a section thickness of $1.25 \mathrm{~mm}$ in 10-mm intervals, and were reviewed in a blinded manner (by ME for the French cohort and by AMHV for the Norwegian cohort), using a simple staging system that was developed by Goh and colleagues (23). Extensive lung disease was defined as $>20 \%$ extent of fibrosis on HRCT or $10-30 \%$ extent of fibrosis and a forced vital capacity (FVC) of $<70 \%$ (23).

PFTs were performed within 1 month of the corresponding $\mathrm{HRCT}$, with results expressed as the percent predicted. A decline in the lung diffusing capacity for carbon monoxide ( $D L_{c o}$ ) of $>15 \%$ and a decline in the FVC of $>10 \%$ were defined as being clinically significant (8). Analyses of the $\mathrm{DL}_{c o}$ were conductedafter exclusion of patients with pulmonary arterial hypertension (PAH). A composite outcome for decline in lung function was created, which included an FVC decline from baseline of $\geq 10 \%$ or an FVC decline of $5-9 \%$ in addition to a $D L_{c o}$ decline of $\geq 15 \%$ (24).

At baseline, the following lung parameters were assessed: 1) presence of lung fibrosis on $H R C T, 2$ ) any lung fibrosis with an FVC of $<70 \%$ predicted, 3) presence of extensive lung disease $(23), 4)$ a $D L_{c o}$ of $<60 \%$ predicted, and 5) correlation with the extent of fibrosis, FVC, and DLco.

For longitudinal analyses, only patients with lung fibrosis at baseline were analyzed. The primary outcome was a decline in the FVC of $>10 \%$ predicted during follow-up. The secondary outcomes were 1) a decline in the FVC of $>10 \%$, with an FVC of $<80 \%$ predicted at follow-up, 2) de novo development of extensive lung disease (23), 3) the composite outcome for lung function decline (24), and 4) a decrease in the $D L_{c o}$ of $>15 \%$. Explanatory analyses involved assessment of the predictive value of each serum biomarker for predicting the risk of death. 
Quantitative analyses of candidate serum biomarkers. Blood samples from both cohorts were obtained with the same standardized procedures, and processing and storing of the blood samples was similar between the Oslo and Paris cohorts. Collection of serum samples occurred a mean \pm SD $0.2 \pm 1.5$ months (range $-6.7,-11.9$ months) before the PFTs and HRCT. Blood was collected during a standard of care blood withdrawal, and then centrifuged at room temperature within 30 minutes. Serum aliquots were stored at $-70^{\circ} \mathrm{C}$ until assayed.

Specific enzyme-linked immunosorbent assay (ELISA) kits were used to analyze the patients' serum for circulating KL-6 (Sekisui Medical), SP-D (DSFPD0; Bio-techne), CCL18 (DCL180B; Bio-techne), and soluble OX40L (CSB-E12752h-5; Clinisciences). According to the manufacturer's protocol, standard samples were run in duplicate, and the coefficients of variation for each respective kit were $<15 \%, 9.3 \%, 9.1 \%$, and $3.4 \%$.

Statistical analysis. Statistical analyses were performed using MedCalc version 12.7. Categorical results were presented as counts and percentages, and continuous variables as the mean $\pm \mathrm{SD}$. Chi-square tests and $t$-tests were used for group comparisons, as appropriate. The accuracy of each serum biomarker for diagnosing the different severities of lung damage at baseline was assessed by receiver operating characteristic $(\mathrm{ROC})$ curve analysis (using the area under the ROC curve $[A \cup C]$ ). The correlation of each biomarker with quantitative values was assessed using Spearman's rho correlation coefficients. High levels of the biomarkers were calculated from ROC curves obtained for the diagnosis of lung fibrosis. The

Table 1. Demographic and clinical characteristics of the patients in each cohort at baseline*

\begin{tabular}{|c|c|c|c|}
\hline Characteristic & $\begin{array}{l}\text { Combined cohort } \\
\qquad(n=427)\end{array}$ & $\begin{array}{l}\text { Norwegian cohort } \\
\qquad(n=262)\end{array}$ & $\begin{array}{l}\text { French cohort } \\
\qquad(n=165)\end{array}$ \\
\hline Male sex & $78 / 427(18.3)$ & $42 / 262(16.0)$ & $36 / 165$ (21.8) \\
\hline Diffuse subtype & $151 / 427(35.4)$ & $80 / 262(30.5)$ & $71 / 165(43)^{\dagger}$ \\
\hline Age, mean \pm SD years & $59.6 \pm 13.6$ & $61.1 \pm 13.6$ & $57.2 \pm 8.5 \ddagger$ \\
\hline Disease duration, mean \pm SD years & $6.2 \pm 6.2$ & $4.3 \pm 2.8$ & $9.3 \pm 8.5 \ddagger$ \\
\hline Anti-topoisomerase I & $101 / 427(23.6)$ & $41 / 262(15.6)$ & $60 / 165(36.4) \ddagger$ \\
\hline ACAs & $181 / 427(42.4)$ & $135 / 262(51.5)$ & 46/165 (27.9)‡ \\
\hline Anti-RNA polymerase III & $33 / 427(7.7)$ & $28 / 262(10.7)$ & $5 / 165(3.0) \ddagger$ \\
\hline Smoking, past or current & $170 / 422(40.3)$ & $116 / 257(45.1)$ & $54 / 165(32.7)^{\dagger}$ \\
\hline Precapillary PH & $39 / 416(9.4)$ & $24 / 251(9.6)$ & $15 / 165(9.1)$ \\
\hline SRC & $18 / 421(4.3)$ & $17 / 256(6.6)$ & $1 / 165(0.6) \ddagger$ \\
\hline Digital ulcers, past or current & $187 / 417(44.8)$ & $114 / 252(45.2)$ & $73 / 165(44.2)$ \\
\hline MRSS, mean \pm SD (no. assessed) & $9.1 \pm 9.5(406)$ & $8.8 \pm 9.1(247)$ & $9.4 \pm 10.2(159)$ \\
\hline MRSS $\geq 14$ & $107 / 406(26.3)$ & $59 / 247(23.9)$ & $48 / 159(30.2)$ \\
\hline Lung fibrosis & $234 / 415(56.4)$ & $149 / 250(59.6)$ & $85 / 165(51.5)$ \\
\hline Extent of lung fibrosis, mean \pm SD \%§ & $14.6 \pm 15.1$ & $10.3 \pm 13.1$ & $22.4 \pm 15.4 \ddagger$ \\
\hline FVC, mean \pm SD $\%$ predicted & $95.5 \pm 21.1$ & $95.3 \pm 19.7$ & $95.8 \pm 23.3$ \\
\hline DLco, mean \pm SD $\%$ predicted & $66.1 \pm 19.7$ & $66.4 \pm 20.1$ & $65.6 \pm 19.1$ \\
\hline Lung fibrosis and FVC $<70 \%$ predicted & $48 / 415$ (11.6) & $27 / 250(10.8)$ & $21 / 165$ (12.7) \\
\hline Extensive disease & $66 / 427(15.5)$ & $30 / 262(11.4)$ & $36 / 165(21.8)$ \\
\hline DLco $<60 \%$ predicted & $119 / 376(31.6)$ & $76 / 238$ (31.9) & $43 / 138(31.2) \dagger$ \\
\hline CCL18 levels, mean \pm SD ng/ml & $67.6 \pm 40.1$ & $69.3 \pm 45.3$ & $64.9 \pm 29.9$ \\
\hline SP-D levels, mean \pm SD $\mathrm{ng} / \mathrm{ml}$ & $15.0 \pm 11.8$ & $15.9 \pm 12.3$ & $13.7 \pm 11.0$ \\
\hline KL-6 levels, mean \pm SD units $/ \mathrm{ml}$ & $963.3 \pm 1,129.9$ & $728 \pm 1,004$ & $1,336 \pm 1,218 \ddagger$ \\
\hline OX40L levels, mean \pm SD ng/ml & $2.8 \pm 2.5$ & $2.4 \pm 2.2$ & $3.5 \pm 2.9 \ddagger$ \\
\hline
\end{tabular}

* Lung fibrosis was diagnosed on high-resolution computed tomography. Precapillary pulmonary hypertension (PH) was diagnosed at the time of right-sided heart catheterization. Except where indicated otherwise, values are the number/total number (\%) of patients. ACAs = anticentromere antibodies; SRC = scleroderma renal crisis; MRSS = modified Rodnan skin thickness score (maximum possible score of 51); FVC = forced vital capacity; SP-D = lung epithelial-derived surfactant protein D; KL-6 = Krebs von den Lungen 6 glycoprotein; OX40L = OX40 ligand.

$\dagger P<0.05$ versus Norwegian cohort.

$\ddagger P<0.01$ versus Norwegian cohort.

§ Extensive disease (\% of affected lung area) in patients with lung fibrosis was defined according to the staging system described by Goh and colleagues (23).

Tा The lung diffusing capacity for carbon monoxide $(\mathrm{D} L \mathrm{c})$ was determined after exclusion of 39 patients with precapillary $\mathrm{PH}$. 
sensitivity, specificity, positive predictive value (PPV), and negative predictive value (NPV) were calculated. Logistic regression analyses, using odds ratios (ORs) with 95\% confidence intervals $(95 \% \mathrm{Cls})$, were carried out to assess the potential risk factors for SSc-associated ILD at baseline, including all factors that yielded significance values of $P$ less than 0.1 in univariate analysis. Multivariate analyses were adjusted for age and disease duration, and significance was determined after Bonferroni correction for multiple comparisons. Following this analysis, we constructed a matrix-based logistic regression to assess the probability of lung fibrosis, and using this matrix model, we were able to stratify the patients into 3 groups of risk (mild, moderate, and high).

The individual value of each biomarker and/or combination of some of these biomarkers was assessed to pre- dict the worsening of ILD. Potential prognostic factors were first analyzed using a Cox proportional hazards regression model (with hazard ratios [HRs]) in single-variable analysis. All factors emerging with significance values of $P$ less than 0.10 by single-variable analysis were included in a multiplevariable model that was adjusted for age and disease dura- tion. Using this model, we built a matrix-based logistic regression in which we assessed patients with ILD at baseline to identify those whose disease would likely progress during the follow-up (25). A $P$ value less than 0.05 was considered statistically significant.

The XLSTAT-Power program was used to estimate the power observations associated with the Cox regression model in the Norwegian cohort, the French cohort, and the combined cohort. The statistical power of this sample was determined, taking into account our sample size, a type I error rate of $5 \%$, and the observed frequency of each outcome.

\section{RESULTS}

Clinical characteristics of the patients. The study cohort comprised 427 patients (262 Norwegian patients from the Oslo University Hospital SSc cohort, and 165 French patients from the Paris Cochin Hospital SSc cohort), including 151 patients (35\%) with diffuse cutaneous SSc. The mean \pm $\mathrm{SD}$ disease duration was $6.2 \pm 6.2$ years. The baseline HRCT showed findings consistent with lung fibrosis in 234 (56.4\%)
A

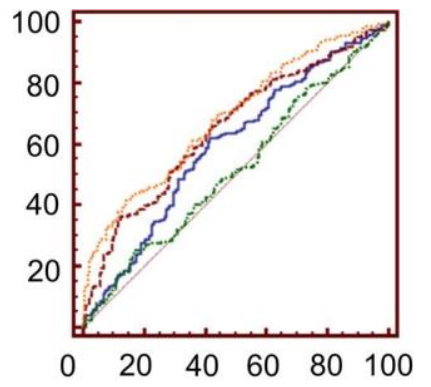

C

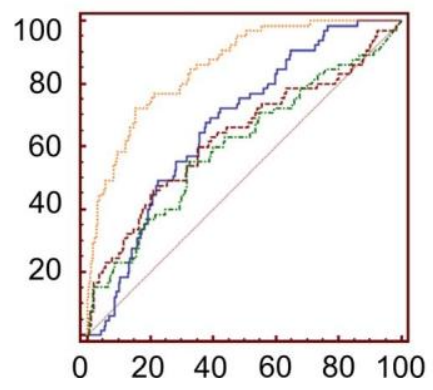

B

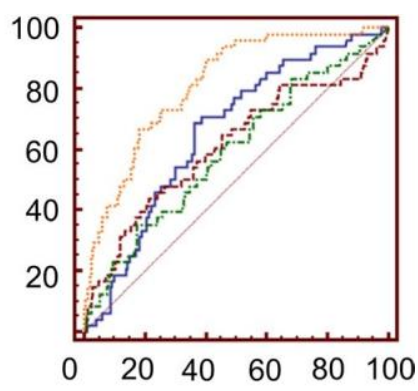

D

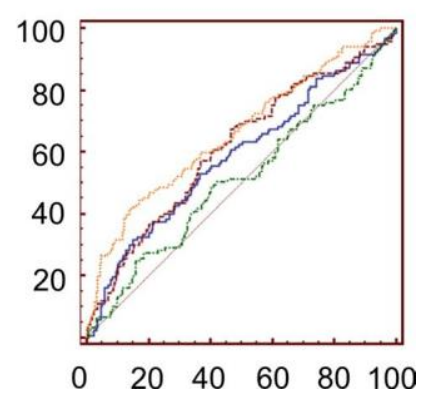

Figure 1. Diagnostic performance of serum levels of Krebs von den Lungen 6 glycoprotein (KL-6) (orange), CCL18 (blue), OX40 ligand (OX40L) (green), and lung epithelial-derived surfactant protein D (SP-D) (brown) for a diagnosis of lung fibrosis on high-resolution computed tomography $(\mathrm{HRCT})(\mathrm{A})$, for severe lung fibrosis (defined as the presence of fibrosis on HRCT and a forced vital capacity of $<70 \%$ predicted) (B), for extensive lung fibrosis (as defined by Goh et al [23]) (C), and for a diffusing capacity for carbon monoxide of $<60 \%$ predicted (after exclusion of 39 patients with pulmonary arterial hypertension) (D) in the combined cohort of patients with systemic sclerosis. Values were calculated using area under the receiver operating characteristic (ROC) curves (AUCs). The x-axes correspond to 100-specificity, and the y-axes correspond to sensitivity. In $A$, for KL-6, AUC 0.689 (95\% confidence interval [95\% Cl] 0.642-0.734) $(P<0.0001)$; for CCL18, AUC 0.592 (95\% CI 0.543-0.640) $(P=0.001)$; for OX40L, AUC 0.521 (95\% Cl 0.471-0.570) ( $P=0.4683)$; and for SP-D, AUC 0.649 (95\% Cl 0.601-0.695) ( $P<0.0001)$. In B, for KL-6, AUC 0.809 (95\% Cl 0.769-0.846) ( $P<0.0001)$; for CCL18, AUC 0.657 (95\% Cl 0.609-0.702) $(P<0.0001)$; for OX40L, AUC 0.590 (95\% Cl 0.542-0.638) $(P=0.0447)$; and for SP-D, AUC $0.613(95 \% \mathrm{Cl} 0.564-0.659)(P=0.0209)$. In C, for KL-6, AUC $0.850(95 \% \mathrm{Cl} 0.813-0.883)(P<0.0001)$; for CCL18, AUC 0.671 (95\% Cl 0.624-0.716) ( $P<0.0001)$; for OX40L, AUC 0.596 (95\% Cl 0.547-0.643) ( $P=0.0195)$; and for SP-D, AUC 0.634 (95\% Cl 0.587-0.680) ( $P=0.0012)$. In D, for KL-6, AUC 0.663 (95\% Cl 0.613-0.711) $(P<0.0001)$; for CCL18, AUC 0.584 (95\% Cl 0.532-0.635) $(P=0.0097)$; for OX40L, AUC 0.517 (95\% Cl 0.465-0.569) ( $P=0.6064)$; and for SP-D, AUC $0.617(95 \%$ Cl 0.566-0.667) $(P=0.0002)$. Color figure can be viewed in the online issue, which is available at http://onlinelibrary.wiley.com/doi/10.1002/art.40815/abstract. 
of the 415 SSc patients assessed. Other characteristics of the cohort at baseline, including the results of PFTs, are summarized in Table 1.

During a mean \pm SD follow-up of $3.2 \pm 4.4$ years, 34 (14.5\%) of 234 patients (having lung fibrosis at baseline) had a decline in the FVC of $>10 \%$, whereas 26 (11\%) developed extensive lung disease de novo. During the follow-up, 93 (39.7\%) of 234 patients with baseline lung fibrosis were treated with immunosuppressants, of whom 45 (48.4\%) received methotrexate, 41 $(44.1 \%)$ received mycophenolate mofetil, 34 (36.6\%) received cyclophosphamide, 31 (33.3\%) received azathioprine, and 25 $(26.9 \%)$ received rituximab, while 2 patients underwent autologous stem cell transplantation. In total, 89 (38.0\%) of the 234 patients with SSc-associated ILD at baseline were treated with steroids during the follow-up.

Assessment of diagnosis of lung fibrosis at baseline. SSc patients with a diagnosis of lung fibrosis could be discriminated on the basis of the baseline serum levels of $\mathrm{KL}$ 6, SP-D, and CCL18, with the best AUCs observed for KL-6 (AUC 0.689, 95\% CI 0.642-0.734; $P<0.001$ ) (best cutoff value 923 units $/ \mathrm{ml}$, with a sensitivity of $44 \%$, specificity of $85 \%$, PPV of $79 \%$, and NPV of $54 \%$ ) and for SP-D (AUC $0.649,95 \% \mathrm{Cl}$ $0.601-0.695 ; P<0.001$ ) (best cutoff value $19.880 \mathrm{ng} / \mathrm{ml}$, with a sensitivity of $36 \%$, specificity of $88 \%$, PPV of $79 \%$, and NPV of $51 \%$ ). With regard to assessment of CCL18 serum levels for the identification of patients with lung fibrosis at baseline, the AUC was $0.592(95 \% \mathrm{Cl} 0.543-0.640 ; P=0.001)$ (best cutoff value $56.6 \mathrm{ng} / \mathrm{ml}$, with a sensitivity of $62 \%$, specificity of $58 \%$, PPV of $66 \%$, and NPV of $54 \%$ ). The ROC curve for the diagnosis of lung fibrosis according to OX40L levels was not statistically significant (Figure 1A).
Using the best cutoff values, we considered the 4 serum protein markers as qualitative variables, and separated patients into high and low levels of each protein. In univariate analyses, all 4 of the biomarkers were associated with lung fibrosis at baseline. In multivariate analyses adjusted for age and disease duration, high serum levels of SP-D (OR 3.15, 95\% CI 1.81-5.48; $P<0.001)$, highserumlevels of KL-6(OR2.41,95\% Cl1.43-4.07; $P=0.001)$, and presence of anti-topoisomerase I antibodies (OR 3.76, 95\% Cl 1.96-7.21; $P<0.001$ ) were independently associated with a diagnosis of lung fibrosis, with the highest weight for the combination of SP-D and anti-topoisomerase I antibodies (Table 2).

We built a matrix logistic regression model based on this analysis, and using this matrix model, we were able to stratify patients into 3 groups of risk for the diagnosis of lung fibrosis according to the baseline serum levels of SP-D and the presence of anti-topoisomerase lantibodies (mild risk, 30-44\%; moderate risk, 45-70\%; and high risk, $\geq 70 \%$ ) (Figure 2A). This model had a sensitivity of $97 \%$, specificity of $69 \%$, PPV of $80 \%$, and NPV of $95 \%$ for the diagnosis of SSc-associated ILD.

Assessment of lung fibrosis severity at baseline. The baseline serum levels of all 4 proteins could identify patients with restrictive lung disease (FVC $<70 \%$ predicted) underlying lung fibrosis, with $\mathrm{KL}-6$ being the strongest biomarker, having an AUC of $0.803(95 \% \mathrm{Cl} 0.769-0.846)(P<0.001)$ (Figure 1B). In univariate analysis, all 4 biomarkers were associated with restrictivelung disease, whereas in multivariate analysis, only KL-6 serum levels (OR 5.24, 95\% Cl 2.61-10.53; $P<0.001$ ) and anti-topoisomerase I antibodies (OR 3.76, 95\% Cl 1.96-7.21; $P<0.001$ ) were significantly associated with restrictive lung disease (Table 2).

Consistently, KL- 6 was the most discriminant biomarker for extensive lung disease (23) (AUC 0.850, 95\% CI 0.813-0.883;

Table 2. Association of biomarkers with lung fibrosis and restrictive lung disease at baseline in multivariate analyses*

\begin{tabular}{|c|c|c|c|c|}
\hline \multirow[b]{2}{*}{ Variable } & \multicolumn{2}{|c|}{ Lung fibrosis } & \multicolumn{2}{|c|}{ Restrictive lung disease } \\
\hline & $P$ & OR $(95 \% \mathrm{Cl})$ & $P$ & OR $(95 \% \mathrm{Cl})$ \\
\hline High KL-6 & 0.001 & $2.411(1.429-4.071)$ & $<0.001$ & $5.243(2.610-10.533)$ \\
\hline High CCL18 & NS & - & NS & - \\
\hline High OX40L & NS & - & NS & - \\
\hline High SP-D & $<0.001$ & 3.153 (1.814-5.479) & NS & - \\
\hline MRSS $\geq 14$ & NS & - & NS & - \\
\hline Anti-topoisomerase I antibodies & $<0.001$ & 3.762 (1.963-7.209) & 0.003 & $2.782(1.406-5.503)$ \\
\hline Male sex & NS & - & 0.036 & - \\
\hline Diffuse cutaneous form of SSc & 0.022 & - & NS & - \\
\hline
\end{tabular}

* Restrictive lung disease in patients with systemic sclerosis (SSc) was defined as the presence of lung fibrosis on high-resolution computed tomography and a forced vital capacity of $<70 \%$ predicted. Variables identified as significant at $P<0.1$ in univariate analysis were tested in multivariate analyses, which were adjusted for age and disease duration. Cutoff values to define high levels of biomarkers were obtained from receiver operating characteristic curves. High serum biomarker levels were defined as follows: for Krebs von den Lungen 6 glycoprotein (KL-6), $\geq 923 \mathrm{IU} / \mathrm{ml}$; for CCL18, $\geq 56.5722 \mathrm{ng} / \mathrm{ml}$; for OX40 ligand (OX40L), $\geq 4.4348$ $\mathrm{ng} / \mathrm{ml}$; for lung epithelial-derived surfactant protein D (SP-D), $\geq 19.880 \mathrm{ng} / \mathrm{ml}$. In multivariate analyses, significance values at $P<0.006$ were obtained after Bonferroni correction for multiple comparisons. $0 R=$ odds ratio; $95 \% \mathrm{Cl}=95 \%$ confidence interval; NS = not significant; MRSS = modified Rodnan skin thickness score. 
A

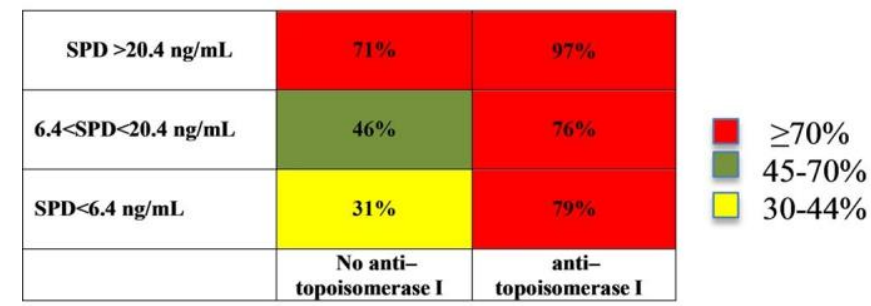

B

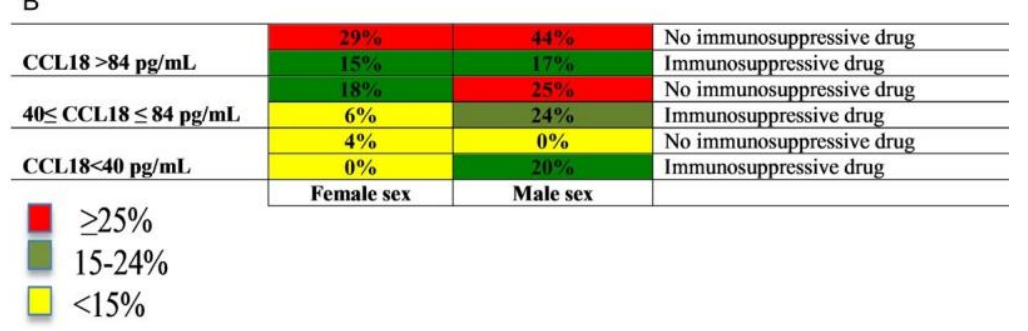

Figure 2. Matrix models. A, Risk of lung fibrosis on high-resolution computed tomography according to lung epithelial-derived surfactant protein D (SP-D) serum levels as well as presence of anti-topoisomerase I antibodies at baseline. Three groups of risk could be identified, stratifying patients as being at high, moderate, or low risk of lung fibrosis. B, Risk of worsening of lung fibrosis during the follow-up in patients with lung fibrosis at baseline, according to CCL18 serum levels, the use of immunosuppressive treatments, and male versus female sex. Three groups of risk could be identified, stratifying patients as being at high, moderate, or low risk of worsening of lung fibrosis. Immunosuppressive drugs correspond to treatment given during the follow-up and include methotrexate, mycophenolate mofetil, azathioprine, cyclophosphamide, anti-tumor necrosis factor, tocilizumab, rituximab, and autologous stem cell transplantation.

$P<0.001$ ) (Figure 1C). In univariate analysis, the 4 serum proteins were associated with extensive lung disease, whereas in multivariate analysis, only associations with $\mathrm{KL}-6$ serum levels (OR8.33, 95\% Cl 4.30-16.14; $P<0.001)$ and anti-topoisomerase I antibodies (OR 2.87, 95\% Cl 1.51-5.45; $P=0.001$ ) were significant.

All 4 of the serum biomarkers could identify patients with a $\mathrm{DL}_{\text {co }}$ of $<60 \%$ predicted (after exclusion of 39 patients with $\mathrm{PAH}$ ), but KL-6 showed the strongestassociation (Figure 1D). In univariate analysis, serum levels of KL-6, SP-D, and CCL18 wereassociated with a $D L_{c o}$ of $<60 \%$ predicted, whereas in multivariate analysis, only the KL-6 serum level was significantly associated with a $\mathrm{DL}_{\text {co }}$ of $<60 \%$ predicted (OR 2.91, 95\% Cl 1.77-4.79; $P<0.001$ ).

All 4 biomarkers correlated with the extent of fibrosis, but the serum levels of $\mathrm{KL}-6$ were the most strongly correlated with the extent of fibrosis $(r=0.551, P<0.001)$ as well as with the FVC $(r=-0.317, P<0.001)$ and the $D_{c o}(r=-0.335, P<$ $0.001)$.

Predictive value of biomarkers in longitudinal analyses of SSc patients having ILD at baseline. Primary outcome. In univariate analyses, only high serum levels of CCL18 were predictive of a decrease in the FVC of $>10 \%$ during the follow-up(Figure 3). In multivariate Coxanalyses adjusted for age and disease duration, high serum levels of CCL18 (HR2.90, 95\% Cl 1.25-6.73; $P=0.01$ ) and male sex (HR 2.48, 95\% Cl 1.165.30; $P=0.02$ ) were independent predictors of a decrease in the FVC of $>10 \%$, whereas the use of immunosuppressive drugs during the follow-up was protective against a decline in the FVC (HR 0.39, 95\% Cl 0.17-0.87; $P=0.02$ ) (Table 3).

Based on these results, we built a matrix model for prognosis. Among SSc patients with ILD at baseline, this prognostic model was able to identify those at low risk $(<15 \%)$, mild risk (15-24\%), or high risk ( $\geq 25 \%$ ) of worsening of lung fibrosis during the follow-up (Figure 2B).

Secondary outcomes. In univariate analysis, CCL-18 serum levels and a modified Rodnan skin thickness score of $\geq 14$ were predictors of a decrease in the FVC of $>10 \%$ and an FVC of $<80 \%$ predicted at follow-up, whereas the use of immunosuppressive drugs was protective (Figure 3B). However, in multivariate analysis, none of the variables showed a significantassociation with the FVC.

In univariate analysis, CCL18 and SP-D serum levels were predictive of the de novo development of extensive disease during the follow-up period (Figure 3C). In multivariate Coxanalysis, only high serum levels of CCL18 (HR 3.71, 95\% Cl 1.02-13.52; $P<0.05)$ and high serum levels of SP-D (HR2.47, 95\% Cl 1.075.71; $P=0.03$ ) were independently predictive of the de novo development of extensive disease, whereas the use of immunosuppressive drugs was protective against extensive disease $(\mathrm{HR}$ 0.33, 95\% Cl 0.12-0.89; $P=0.03$ ) (Table 3).

In multivariate analyses, only high serum levels of CCL18 at baseline predicted the composite outcome of lung function decline (Figure 3D) (24) and decrease in the DLco of $>15 \%$ (after exclusion of 21 patients with precapillary pulmonary hypertension) during the follow-up (HR 3.00, 95\% Cl 1.43-6.28 [P<0.01] and 
A

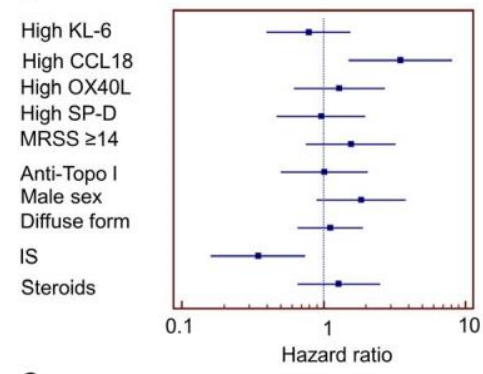

C

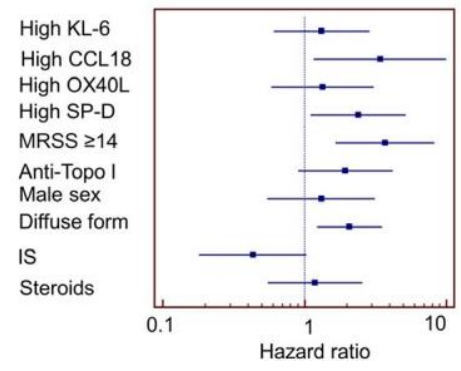

B

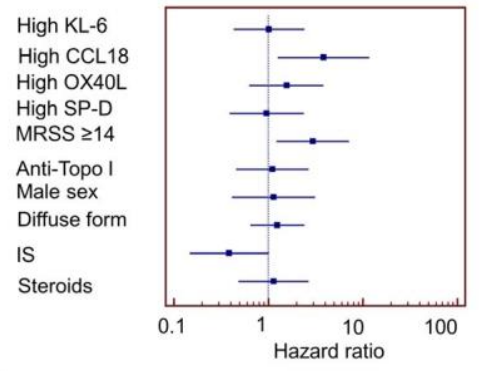

D

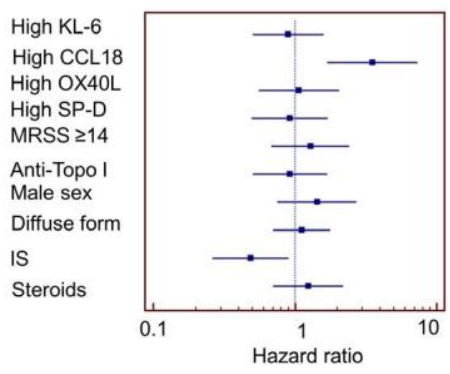

Figure 3. Forest plots from Cox univariate analyses analyzing the predictive value of the serum biomarkers and other variables for worsening of lung fibrosis, using the following outcomes: a decrease in the forced vital capacity (FVC) of $>10 \%$ predicted (A), a decrease in the FVC of $>10 \%$ predicted and an FVC at follow-up of $<80 \%$ predicted (B), de novo development of extensive disease (C), and development of a composite outcome (D). Solid squares with bars represent the hazard ratios with $95 \%$ confidence intervals. Extensive disease, according to that described by Goh and colleagues, was defined as extent of fibrosis $>20 \%$ on high-resolution computed tomography or extent of fibrosis $10-30 \%$ and an FVC of $<70 \%$ predicted (23). A composite outcome for lung function decline was defined as an FVC decline from baseline of $\geq 10 \%$ or an FVC decline of $5-9 \%$ in addition to a decline in the diffusing capacity for carbon monoxide of $\geq 15 \%$ (24). Immunosuppressive (IS) drugs were those given during the follow-up and included methotrexate, mycophenolate mofetil, azathioprine, cyclophosphamide, anti-tumor necrosis factor, tocilizumab, rituximab, and autologous stem cell transplantation. KL-6 = Krebs von den Lungen 6 glycoprotein; OX40L = OX40 ligand; SP-D = lung epithelial-derived surfactant protein D; MRSS = modified Rodnan skin thickness score; anti-Topo I = anti-topoisomerase I antibodies. Color figure can be viewed in the online issue, which is available at http://onlinelibrary.wiley.com/doi/10.1002/art.40815/abstract.

HR3.00, 95\% Cl1.27-7.11 $[P=0.01])$. No biomarker was predictive of short-term (1-year) lung fibrosis worsening.

In patients who had lung fibrosis at baseline and who received immunosuppressive drugs during the follow-up, 9 (9.9\%) of 91 displayed a worsening of ILD. There was a trend toward a worse ILD outcome in patients who had high baseline serum levels of CCL-18, but this did not reach significance (HR2.39, 95\% Cl 0.60-9.60; $P=0.22$ ).

Explanatory analyses. In total, 53 deaths (22.6\% of patients) were recorded in the group of SSc patients with ILD. None of the 4 biomarkers was predictive of death.

\section{DISCUSSION}

The results of our study have highlighted the respective positioning of these 4 candidate serum biomarkers in patients with SSc-associated ILD, in which the SP-D serum level appears to be the best biomarker for the diagnosis of SSc-associated ILD, whereas $\mathrm{KL}-6$ serum levels are discriminant for measuring the severity of SSc-associated ILD. In longitudinal analyses, CCL18 serum levels appeared to be a strong prognostic marker for progression of SSc-associated ILD.
A biomarker is "a characteristic objectively measured and evaluated as an indicator of normal biological processes, pathogenic processes, or pharmacologic responses to a therapeutic intervention" (2). Biomarkers can be divided into 3 categories of utility: diagnostic, prognostic (predicting the development of a complication), and predictive (predictive of the response to a therapy). In our study, the serum level of SP-D was a strong biomarker for the diagnosis of SSc-associated ILD. Indeed, by combining SP-D serum levels with the presence of anti-topoisomerase I antibodies, we could accurately discriminate, at baseline, 3 distinct groups of patients at risk of lung fibrosis, with a sensitivity of $97 \%$, specificity of $69 \%$, PPV of $80 \%$, and NPV of $95 \%$. $\mathrm{KL}-6$ was identified as a strong biomarker for staging lung fibrosis severity, with a performance that was superior to that of clinical characteristics of the disease, antibodies, and other biomarkers. Interestingly, we observed moderate-to-high correlations between $\mathrm{KL}-6$ serum levels and the FVC, DLco, and extent of fibrosis on HRCT, in accordance with previous studies (25-28).

Taken together, these results hold great promise for early detection of SSc patients who might be considered at high risk of developing ILD by using systematic measurement of SP-D serum levels and autoantibodies at the time of SSc diagnosis, whereas serum levels of KL-6 could be used to assess ILD severity. In the 
Table 3. Factors predictive of worsening of lung fibrosis during the follow-up in multivariate Cox analysis

\begin{tabular}{|c|c|c|c|c|}
\hline \multirow[b]{2}{*}{ Variable } & \multicolumn{2}{|c|}{ Decrease in $\mathrm{FVC}>10 \%$} & \multicolumn{2}{|c|}{ Extensive disease de novo } \\
\hline & $P$ & $\mathrm{HR}(95 \% \mathrm{Cl})$ & $P$ & $\mathrm{HR}(95 \% \mathrm{Cl})$ \\
\hline High KL-6 & NS & - & NS & - \\
\hline High CCL18 & 0.014 & $2.901(1.250-6.735)$ & 0.048 & $3.712(1.019-13.522)$ \\
\hline High OX40L & NS & - & NS & - \\
\hline High SP-D & NS & - & 0.035 & $2.470(1.068-5.713)$ \\
\hline MRSS $\geq 14$ & NS & - & NS & - \\
\hline Anti-topoisomerase I antibodies & NS & - & NS & - \\
\hline Male sex & 0.019 & - & NS & - \\
\hline Diffuse cutaneous form of SSC & NS & $2.485(1.165-5.298)$ & NS & - \\
\hline Immunosuppressive drugs & 0.022 & $0.387(0.173-0.869)$ & 0.029 & $0.329(0.122-0.887)$ \\
\hline Steroids & NS & - & NS & - \\
\hline
\end{tabular}

* Variables identified as significant at $P<0.1$ in Cox univariate analysis were tested in multivariate analyses, which were adjusted for age and disease duration. The analysis included only systemic sclerosis (SSc) patients with lung fibrosis at baseline. Extensive disease, according to the staging system of Goh and colleagues (23), was defined as extent of fibrosis $>20 \%$ on high-resolution computed tomography or extent of fibrosis $10-30 \%$ and a forced vital capacity (FVC) of $<70 \%$ predicted (23). Cutoff values to define high levels of biomarkers were obtained from receiver operating characteristic curves for diagnosis of lung fibrosis. High serum biomarker levels were defined as follows: for Krebs von den Lungen 6 glycoprotein (KL-6), $\geq 923$ IU/ml; for CCL18, $\geq 56.5722 \mathrm{ng} / \mathrm{ml}$; for $0 X 40$ ligand (OX40L), $\geq 4.4348 \mathrm{ng} / \mathrm{ml}$; for lung epithelial-derived surfactant protein $D$ (SP-D), $\geq 19.880 \mathrm{ng} / \mathrm{ml}$. Immunosuppressive drugs correspond to treatment given during the follow-up, including methotrexate, mycophenolate mofetil, azathioprine, cyclophosphamide, anti-tumor necrosis factor, tocilizumab, rituximab, and autologous stem cell transplantation. HR = hazard ratio; $95 \%$ $\mathbf{C I}=\mathbf{9 5} \%$ confidence interval; NS = not significant; MRSS = modified Rodnan skin thickness score.

future, this could help determine whether or not HRCT should be performed in an individual SSc patient according to the patient's specific risk of lung fibrosis. This early risk stratification might avoid unnecessary and expensive radiating imaging.

The ultimate goal in utilizing a biomarker is to improve the patient's prognosis, thereby leading to the development of personalized medicine, which is a crucial unmet need in a heterogeneous disease such as SSc. The results of our study validate the CCL-18 serum level as a strong prognostic biomarker of the worsening of SSc-associated ILD over a 3-year period $(15,19,22,29-31)$. Previously, small-sized studies from Franceand Germany showed thathigh CCL18serumlevels were associated with a decrease of $>10 \%$ in the FVC over 4 years and 2 years, respectively $(30,31)$. More recently, we observed that high CCL18 serum levels were predictive of a decline in the FVC of $>10 \%$ (22). Ourstudy strengthens these previous findings throughalargersamplesizewithmoreaccurateanalyses, including multivariate analyses in which other clinical and biologic data as well as other relevant ILD biomarkers were taken into account.

Of most interest, unlike previous studies, we restricted our longitudinal analysis to patients whose HRCT had already shown evidence of ILD at baseline $(22,29,31,32)$. Indeed, there is a crucial need to identify, at the time of diagnosis, those SSc patients with ILD whose disease will rapidly progress to an extensive fibrosis. Herein, our study showed that high serum levels of CCL18 multiplied by 3 the risk of a 10\% decrease in the FVC in patients with SSc-associated ILD. To better help clinicians in delineating the prognosis in their patients, we constructed a matrix model based on the presence of ILD at baseline, which showed that female patients with low serum levels of CCL18 had a very low risk of lung fibrosis worsening during the follow-up, whereas male patients with CCL 18 serum levels of more than $84 \mathrm{pg} / \mathrm{ml}$ were at high risk of lung fibrosis worsening. Thus, measurement of the CCL-18 serum level at baseline could help in risk stratification of SSc patients with ILD and should lead to close monitoring of highrisk patients (male, highserum levels of CCL18) by repeated PFTs and HRCT.

In the US Genetics versus Environment in Scleroderma Outcome Study (GENISOS), comprising 266 patients with SSc, the serum level of CCL18 was a predictor of short-term (1-year) decline in the FVC, but not over the longer term (32). Some differences between our study and the GENISOS study may be explained by the following discrepancies. The GENISOS cohort included a high proportion of African American patients and $20 \%$ of the cohort were positive for anti-RNA polymerase III antibodies, whereas our study included only Caucasian patients and $8 \%$ of the patients had anti-RNA polymerase III antibodies. Furthermore, in the GENISOS study, both controls and patients had high serum levels of CCL18 ( 2-fold over our cutoff value), which raises the question as to whether there were confounding factors responsible for the increase in CCL18 levels (32).

In our study, immunosuppressive drugs reduced lung fibrosis worsening in SSc patients who had ILD at baseline. When considering only patients with SSc-associated ILD who received immunosuppressive drugs, there was a trend toward worse lung outcomes in patients with high baseline serum CCL18 
levels, but this did not reach significance $(P=0.22)$, probably because of the low number of events (9 of 91 ). Our study was conducted in 2 observational cohorts in which treatment regimens were heterogeneous, although we tried to address this limitation by adjusting for immunosuppressive agents in the multivariate analyses. Therefore, this study was not well-suited for developing predictive biomarkers for response to specific therapeutic agents.

Besides their clinical use, biomarkers are also relevant for the elucidation of disease mechanisms. KL-6 is expressed mainly on type II pneumocytes in alveoli and bronchiolar epithelial cells, particularly on proliferating and regenerating type II pneumocytes, and an increase in KL-6 levels in severe lung disease might reflect regeneration of lung epithelium secondary to lung damage. CCL18 is constitutively produced by antigen-presenting cells, particularly by dendritic cells and macrophages in lung tissue, and is highly inducible by inflammatory stimuli. M2 macrophages are known to release CCL18, which attracts immune cells and was shown to directly stimulate collagen production in fibroblasts, contributing to worsening of fibrosis $(33,34)$. Thus, one might speculate that high CCL18 levels in the serum could reflect the activation status of M2 macrophages in ILD-affected lung tissue, providing potential links between M2 macrophages, inflammation, and fibrosis and the pathogenesis of SSc-associated ILD. Our findings also support the identification of CCL18 as a potential therapeutic target.

To our knowledge, our study is the largest study of potential lung biomarkers in SSc to date with prospective standardized lung assessment, comparing concomitantly 4 of the most promising ILD markers and including both HRCT and PFT data as well as data from multivariate analyses. Furthermore, only 22 patients (5\%) of the cohort were lost to follow-up.

Although the serum level of SP-D was a strong predictor of lung fibrosis at baseline, in longitudinal analyses, SP-D was not predictive of lung fibrosis worsening in most of the analyses. These results are not consistent with those of previous studies $(35,36)$, but those earlier studies were small-sized, diagnosed lung fibrosis by radiography rather than HRCT, included both mixed connective tissue diseases and SSc, and did not perform multivariate analyses.

We previously highlighted OX40L both as an important mechanistic molecule and as a candidate biomarker in lung fibrosis (19). Herein in the multivariate analyses, we did not confirm these previous results with regard to $\mathrm{OX} 40 \mathrm{~L}$, although a significant association with lung fibrosis was observed in some univariate analyses (19).

In view of the potential clinical use of biomarkers, it is established that a biomarker assay should only be used to guide management if it has 1) analytic validity, 2) clinical validity, and 3) clinical utility $(37,38)$. Analytic validity implies that the test for the biomarker is accurate, reproducible, and reliable. This condition was fulfilled for the biomarkers discussed herein, since commer- cial ELISA kits were available for use. Clinical validity of the biomarkers was also demonstrated, since the serum levels of SP-D and CCL18 (incombination with biologic and clinical data) divided our cohort into 3 distinct risk groups for SSc-associated ILD diagnosis and prognosis. Clinical utility, which implies that use of these biomarker tests would result in better outcomes, or in similar outcomes with less cost, should be studied further, and the sensitivity of the biomarkers to changes (since only baseline serum levels were available) should also be evaluated. Furthermore, the predictive value of our biomarkers for monitoring the clinical response to a treatment should be assessed.

Our study has some limitations. The mean disease duration was 6 years, and therefore the results may not apply to patients with a longer disease duration. It is important to note that in 2 key clinical trials investigating patients with SSc-associated ILD, the patients were recruited when disease duration was $<7$ years $(39,40)$. Indeed, patients with earlier disease of more recentonset are those with the highest risk of progression; such patients might benefit from early risk stratification as a strategy to adapt the management of their disease.

Furthermore, rates of disease progression were low in both cohorts. Therefore, we combined the 2 cohorts to increase the number of events and the power of the study.

SSc-associated ILD in the French patients was characterized by amoreseverephenotype (more diffuseforms, higherfrequency of anti-topoisomerase I antibodies, higher extent of lung fibrosis on CT scan) compared to the Norwegian cohort. However, both cohorts were investigated in a similar manner.

In addition, the current project is an extension of our previous study (19), from which we learned that the number of events and the statistical power is key for investigating ILD in SSc. When the cohorts were analyzed separately, the SP-D serum level was independently associated with lung fibrosis, and the KL-6 serum level was associated with severity of lung fibrosis in both cohorts (data not shown). However, in the longitudinal part of the study, the results were not consistent with regard to the prognostic value of the biomarkers in each independent cohort, mostly likely due to the low number of events and the lack of statistical power. It was previously shown that baseline C-reactive protein (CRP) levelswere predictive of long-term ILD progression (41). However, CRP was not examined as a biomarker in the present study, because CRP data were not available from a sufficient number of patients.

We could not compare the value of our biomarkers in SScassociated ILD with that in other diseases that present with lung fibrosis. However, the goal of the present study was more to improve SSc care rather than to validate lung fibrosis biomarkers that could be used in any context.

Finally, since both cohorts included mainly Caucasians, our results cannot be extrapolated to other racial groups.

To conclude, the serum level of SP-D was identified as a useful biomarker for early identification of SSc patients having 
developed ILD, whereas the serum level of KL-6 was the best biomarker for assessing severity of ILD. CCL18 serum levels could help in risk stratification for lung fibrosis worsening in SSc patients having lung fibrosis at baseline.

Our risk models for the diagnosis and prognosis of SScassociated ILD might help physicians optimize the management of their patient care, since these models could be used as an indication to perform HRCT (according to SP-D serum levels and presence of anti-topoisomerase I antibodies), to increase the frequency of ILD assessment over follow-up (according to CCL-18 serum levels), and perhaps, in the future, to optimize treatment strategies (such as identification of high-risk patients who might benefit from treatment or development of personalized therapies according to predictive biomarkers). Furthermore, our study findings should facilitate cohort enrichment in future clinical trials assessing lung therapies in SSc.

\section{AUTHOR CONTRIBUTIONS}

All authors were involved in drafting the article or revising it critically for important intellectual content, and all authors approved the final version to be published. Dr. Allanore had full access to all of the data in the study and takes responsibility for the integrity of the data and the accuracy of the data analysis.

Study conception and design. Elhai, Hoffmann-Vold, Avouac, Molberg, Allanore.

Acquisition of data. Elhai, Hoffmann-Vold, Pezet, Cauvet, Leblond, Fretheim, Garen, Kuwana.

Analysis and interpretation of data. Elhai, Hoffmann-Vold, Avouac, Allanore.

\section{REFERENCES}

1. Mura M, Porretta MA, Bargagli E, Sergiacomi G, Zompatori M, Sverzellati N, et al. Predicting survival in newly diagnosed idiopathic pulmonary fibrosis: a 3-year prospective study. Eur Respir J 2012;40:101-9.

2. Lake F, Proudman S. Rheumatoid arthritis and lung disease: from mechanisms to a practical approach. Semin Respir Crit Care Med 2014;35:222-38.

3. Tyndall AJ, Bannert B, Vonk M, Airò P, Cozzi F, Carreira PE, et al. Causes and risk factors for death in systemic sclerosis: a study from the EULAR Scleroderma Trials and Research (EUSTAR) database. Ann Rheum Dis 2010;69:1809-15.

4. Elhai M, Meune C, Boubaya M, Avouac J, Hachulla E, Balbir-Gurman A, et al. Mapping and predicting mortality from systemic sclerosis. Ann Rheum Dis 2017;76:1897-1905.

5. Molberg $\varnothing$, Hoffmann-Vold AM. Interstitial lung disease in systemic sclerosis: progress in screening and early diagnosis. Curr Opin Rheumatol 2016;28:613-8.

6. Hoffmann-Vold AM, Aaløkken TM, Lund MB, Garen T, Midtvedt $\varnothing$, Brunborg C, et al. Predictive value of serial high-resolution computed tomography analyses and concurrent lung function tests in systemic sclerosis. Arthritis Rheumatol 2015;67:2205-12.

7. Sgalla G, Biffi A, Richeldi L. Idiopathic pulmonary fibrosis: diagnosis, epidemiology and natural history. Respirology 2016;21:427-37.

8. SilverKC, Silver RM. Management of systemic-sclerosis-associated interstitial lung disease. Rheum Dis Clin North Am 2015;41:439-57.

9. Clarke DL, Murray LA, Crestani B, Sleeman MA. Is personalised medicine the key to heterogeneity in idiopathic pulmonary fibrosis? Pharmacol Ther 2017;169:35-46.
10. Sullivan KM, Goldmuntz EA, Keyes-Elstein L, McSweeney PA, Pinckney A, Welch B, et al. Myeloablative autologous stem- cell transplantation for severe scleroderma. $N$ Engl J Med 2018;378:35-47.

11. Bonella F, Caramaschi P. The ambitious goal of validating prognostic biomarkers for systemic sclerosis-related interstitial lung disease. J Rheumatol 2013;40:1034-6.

12. Affandi AJ, Radstake TR, MarutW. Update on biomarkers in systemic sclerosis: tools for diagnosis and treatment. Semin Immunopathol 2015;37:475-87.

13. Hamai K, Iwamoto H, Ishikawa N, Horimasu Y, Masuda T, Miyamoto S, et al. Comparative study of circulating MMP-7, CCL18, KL-6, SP$A$, and SP-D as disease markers of idiopathic pulmonary fibrosis. Dis Markers 2016;2016:4759040.

14. Zhang $Y$, Kaminski N. Biomarkers in idiopathic pulmonary fibrosis. Curr Opin Pulm Med 2012;18:441-6.

15. Prasse A, Pechkovsky DV, Toews GB, Schäfer M, Eggeling S, Ludwig C, et al. CCL18 as an indicator of pulmonary fibrotic activity in idiopathic interstitial pneumonias and systemic sclerosis. Arthritis Rheum 2007;56:1685-93.

16. Ohshimo S, Ishikawa N, Horimasu Y,Hattori N, Hirohashi N, Tanigawa $\mathrm{K}$, et al. Baseline $\mathrm{KL}-6$ predicts increased risk for acute exacerbation of idiopathic pulmonary fibrosis. Respir Med 2014;108:1031-9.

17. Sato S, Hanibuchi M, Fukuya A, Yabuki Y, Bando H, Yoshijima T, etal. Idiopathic pleuroparenchymal fibroelastosis is characterized by an elevated serum level of surfactant protein-D, but not Krebs von den Lungen-6. Lung 2014;192:711-7.

18. Barlo NP, van Moorsel CH, Ruven HJ, Zanen P, van den Bosch JM, Grutters JC. Surfactant protein-D predicts survival in patients with idiopathic pulmonary fibrosis. Sarcoidosis Vasc Diffuse Lung Dis 2009;26:155-61.

19. Elhai M, Avouac J, Hoffmann-Vold AM, Ruzehaji N, Amiar O, Ruiz B et al. OX40L blockade protects against inflammation-driven fibrosis. Proc Natl Acad Sci U S A 2016;113:E3901-10.

20. Van den Hoogen F, Khanna D, Fransen J, Johnson SR, Baron M, Tyndall A, et al. 2013 classification criteria for systemic sclerosis: an American College of Rheumatology/European League Against Rheumatism collaborative initiative. Arthritis Rheum 2013;65:2737-47.

21. Herzog EL, Mathur A, Tager AM, Feghali-Bostwick C, Schneider F, Varga J. Interstitial lung disease associated with systemic sclerosis and idiopathic pulmonary fibrosis: how similar and distinct? Arthritis Rheumatol 2014;66:1967-78.

22. Hoffmann-Vold AM, Tennøe AH, Garen T, Midtvedt $\varnothing$, Abraityte A, Aaløkken TM, et al. High level of chemokine CCL18 is associated with pulmonary function deterioration, lung fibrosis progression, and reduced survival in systemic sclerosis. Chest 2016;150:299306.

23. Goh NS, Desai SR, Veeraraghavan S, Hansell DM, Copley SJ, Maher TM, et al. Interstitial lung disease in systemic sclerosis: a simple staging system. Am J Respir Crit Care Med 2008;177:124854.

24. Goh NS, Hoyles RK, Denton CP, Hansell DM, Renzoni EA, Maher $\mathrm{TM}$, et al. Short-term pulmonary function trends are predictive of mortality in interstitial lung disease associated with systemic sclerosis. Arthritis Rheumatol 2017;69:1670-8.

25. Visser K, Goekoop-Ruiterman YP, de Vries-Bouwstra JK, Ronday HK, Seys PE, Kerstens PJ, et al. A matrix risk model for the prediction of rapid radiographic progression in patients with rheumatoid arthritis receiving different dynamic treatment strategies: post hoc analyses from the BeSt study. Ann Rheum Dis 2010;69:1333-7.

26. Yanaba K, Hasegawa M, Takehara K, Sato S. Comparative study of serum surfactant protein-D and KL-6 concentrations in patients with systemic sclerosis as markers for monitoring the activity of pulmonary fibrosis. J Rheumatol 2004;31:1112-20. 
27. Hant FN, Ludwicka-Bradley A, Wang HJ, Li N, Elashoff R, Tashkin DP, et al. Surfactant protein D and KL-6 as serum biomarkers of interstitial lung disease in patients with scleroderma. J Rheumatol 2009;36:773-80.

28. Bonella F, Volpe A, Caramaschi P, Nava C, Ferrari P, Schenk K, et al. Surfactant protein D and KL-6 serum levels in systemic sclerosis: correlation with lung and systemic involvement. Sarcoidosis Vasc Diffuse Lung Dis 2011;28:27-33.

29. Kodera M, Hasegawa M, Komura K, Yanaba K, Takehara K, Sato S. Serum pulmonary and activation-regulated chemokine/ CCL18 levels in patients with systemic sclerosis: a sensitive in- dicator of active pulmonary fibrosis. Arthritis Rheum 2005;52: 2889-96.

30. Tiev KP, Hua-Huy T, Kettaneh A, Gain M, Duong-Quy S, Tolédano C, et al. Serum CC chemokine ligand-18 predicts lung disease worsening in systemic sclerosis. Eur Respir J 2011;38:1355-60.

31. Schupp J, Becker M, Günther J, Müller-Quernheim J, Riemekasten G, Prasse A. Serum CCL18 is predictive for lung disease progression and mortality in systemic sclerosis. Eur Respir J 2014;43:1530 2.

32. Elhaj M, Charles J, Pedroza C, Liu X, Zhou X, Estrada-Y-Martin RM, et al. Can serum surfactant protein D or CC-chemokine ligand 18 predict outcome of interstitial lung disease in patients with early systemic sclerosis? J Rheumatol 2013;40:1114-20.

33. Atamas SP, Luzina IG, Choi J, Tsymbalyuk N, Carbonetti NH, Singh IS, et al. Pulmonary and activation-regulated chemokine stimulates collagen production in lung fibroblasts. Am J Respir Cell Mol Biol 2003;29:743-9.

34. Schraufstatter I, Takamori H, Sikora L, Sriramarao P, DiScipio RG. Eosinophils and monocytes produce pulmonary and activation- regulated chemokine, which activates cultured monocytes/macrophages. Am J Physiol Lung Cell Mol Physiol 2004;286:L494-501.

35. Asano Y, Ihn H, Yamane K, Yazawa N, Kubo M, Fujimoto M, et al. Clinical significance of surfactant protein $\mathrm{D}$ as a serum marker for evaluating pulmonary fibrosis in patients with systemic sclerosis. Arthritis Rheum 2001;44:1363-9.

36. YamakawaH, HagiwaraE, KitamuraH, Yamanaka Y, IkedaS, Sekine A, et al. Serum KL-6 and surfactant protein-D as monitoring and predictive markers of interstitial lung disease in patients with systemic sclerosis and mixed connective tissue disease. J Thorac Dis 2017;9:362-71.

37. Hayes DF.Biomarkervalidationandtesting. MolOncol2015;9:960-6.

38. Teutsch SM, Bradley LA, Palomaki GE, Haddow JE, Piper M, Calonge N, et al. The Evaluation of Genomic Applications in Practice and Prevention (EGAPP) Initiative: methods of the EGAPP Working Group. Genet Med 2009;11:3-14.

39. Distler O, Brown KK, Distler JH, Assassi S, Maher TM, Cottin V, et al, on behalf of the SENSICS ${ }^{\mathrm{TM}}$ trial investigators. Design of a randomised, placebo-controlled clinical trial of nintedanib in patients with systemic sclerosis-associated interstitial lung disease (SENSCIS $^{\mathrm{TM}}$ ). Clin Exp Rheumatol 2017;35 Suppl 106:75-81.

40. Tashkin DP, Roth MD, Clements PJ, Furst DE, Khanna D, Kleerup $E C$, et al. Mycophenolate mofetil versus oral cyclophosphamide in scleroderma-related interstitial lung disease (SLS II): a randomised controlled, double-blind, parallel group trial. Lancet Respir Med 2016;4:708-19.

41. Liu X, Mayes MD, Pedroza C, Draeger HT, Gonzalez EB, Harper BE, et al. Does C-reactive protein predict the long-term progression of interstitial lung disease and survival in patients with early systemic sclerosis? Arthritis Care Res (Hoboken) 2013;65:1375-80. 